\title{
Chemotherapeutic Topical Agent
}

National Cancer Institute

\section{Source}

National Cancer Institute. Chemotherapeutic Topical Agent. NCI Thesaurus. Code C2252.

An agent applied topically that inhibits the maturation, growth or spread of tumor cells. 